\title{
CIÊNCIA'NATURA
}

\section{Uma comparação dos erros de estimativa da taxa de dissipação de energia cinética da turbulência por diferentes métodos na camada-limite atmosférica}

\begin{abstract}
A comparison of errors in the turbulence kinetic energy dissipation-rate estimations through different methods in the atmospheric boundary layer
\end{abstract}

Lívia Souza Freire, Nelson Luís Dias e André Luiz Grion

Universidade Federal do Paraná , Brasil

\begin{abstract}
Resumo
Na atmosfera, a taxa de dissipação da energia cinética da turbulência $(\varepsilon)$ pode ser estimada indiretamente a partir da velocidade do vento longitudinal medida em alta frequência, utilizando as equações previstas por Kolmogorov para a faixa inercial do espectro, ogiva ou função estrutura de segunda ordem. Para estimar o erro inerente a essas estimativas, foram criados espectros sintéticos a partir do espectro teórico com a adição de erros aleatórios, utilizados posteriormente na obtenção de ogivas e funções estrutura. A estimativa de $\varepsilon$ a partir dos espectros ou função estrutura apresentou valor de raiz do erro médio quadrático igual a aproximadamente $2 \%$ do valor esperado de $\varepsilon$, enquanto que a estimativa utilizando a ogiva apresentou erro de aproximadamente 1,3\%. É necessário acrescentar um fator de correção das estimativas utilizando a ogiva ou função estrutura, para que seja eliminado o viés causado pela frequência de Nyquist finita. Por apresentar erros menores, o uso da ogiva é o mais apropriado na estimativa do valor de ع na camada-limite atmosférica.
\end{abstract}

Palavras-chave: Espectro, função estrutura, Monte Carlo, ogiva, função estrutura.

Abstract

The dissipation rate of the turbulence kinetic energy $(\varepsilon)$ in the atmosphere can be indirectly obtained from high-frequency measurements of streamwise wind velocity, when using the inertial subrange theory predicted by Kolmogorov for the spectrum, second order structure function or ogive. To estimate the error intrinsic to these estimations, a series of synthetic spectra was constructed from the theoretical spectrum combined with random errors, which were then used to obtain structure functions and ogive. The $\varepsilon$ estimation from spectra or structure functions presented a root mean square value of approximately $2 \%$ of the expected value of $\varepsilon$, whereas the estimation from ogives had errors of approximately $1.3 \%$. A correction factor is needed in the estimations using the ogive and the structure function in order to remove the bias caused by the finite Nyquest frequency. Due to the smaller error, the ogive provides the best approach for indirect estimation of $\varepsilon$ in the atmospheric boundary layer.

Keywords: Dissipation rate, Monte Carlo, ogive, spectrum, structure function. 


\section{Introdução}

O balanço de energia cinética da turbulência (ECT) é parte fundamental dos estudos sobre a dinâmica de escoamentos turbulentos, pois representa o conjunto de processos físicos relevantes para o surgimento e manutenção da turbulência, incluindo a produção, o transporte e a dissipação de energia. Em particular, a taxa de dissipação da ECT $(\varepsilon)$ é uma variável de suma importância por estar presente em todos os escoamentos turbulentos, além de possuir características universais que são extremamente úteis para o entendimento da física da turbulência. Um exemplo claro da importância de $\varepsilon$ consiste na existência da chamada cascata de energia, caracterizada por uma transferência de ECT das maiores para as menores escalas do escoamento sem perda significativa de energia no processo, fenômeno que ocorre em uma faixa de escalas conhecida por faixa inercial. Devido ao fato de que é apenas nas menores escalas do escoamento que a ECT é transformada em energia interna, em um escoamento estacionário a taxa de transferência de energia ao longo da cascata é igual à taxa de dissipação, o que torna $\varepsilon$ o principal parâmetro de caracterização da turbulência. O conceito de cascata de energia, combinado à hipótese de isotropia das menores escalas do escoamento, permitiu que, em 1941, Kolmogorov desenvolvesse leis que governam a faixa inercial, leis essas que são caracterizadas somente pelo valor de $\varepsilon$, e que são consideradas um dos principais (senão o principal) resultado existente na teoria da turbulência (Davidson, 2004).

Um segundo exemplo, de cunho mais prático, é o uso de $\varepsilon$ na definição de escalas de comprimento em escoamentos de camada limite. Trabalhos recentes (Davidson e Krogstad, 2014; Pan e Chamecki, 2016; Chamecki et al., 2017) mostram que a escala formada por $u_{*}$ (velocidade de atrito) e $\varepsilon$ é mais apropriada que a distância da parede na adimensionalização de diferentes tipos de escoamentos (como a camada-limite suave ou rugosa e a camada superficial da atmosfera com ou sem vegetação). Além disso, uma série de modelos de fechamento de escoamentos turbulentos necessitam de estimativas ou parametrizações de $\varepsilon$ (Moeng e Wyngaard, 1989; Katul et al., 2004).

Apesar de sua relevância, a estimativa de $\varepsilon$ ainda representa um desafio em estudos de turbulência atmosférica. Devido à dificuldade em sua medição direta, estimativas indiretas são comumente obtidas a partir de espectros ou funções estrutura (Chamecki e Dias, 2004; Chamecki et al., 2017), utilizando as leis da faixa inercial previstas por Kolmogorov. Entretanto, diferenças nos valores obtidos quando utilizados métodos distintos no mesmo conjunto de dados (Chamecki e Dias, 2004) indicam que as estimativas apresentam uma incerteza significativa. Uma terceira alternativa proposta por Dias (2017) é o uso da ogiva gerada a partir da integração do espectro da velocidade longitudinal. No presente estudo, é investigada a existência de viés e o valor do erro aleatório da estimativa de $\varepsilon$ quando utilizados o espectro, a ogiva ou a função estrutura.

\section{Métodos}

\section{Teoria de Kolmogorov para o espectro, ogiva e função estrutura}

A taxa de dissipação da energia cinética da turbulência em escoamentos incompressíveis pode ser escrita como $\varepsilon=2 \nu \overline{s_{i j} s_{i j}}$, onde $\nu$ é a viscosidade molecular do fluido e $s_{i j}$ é o tensor taxa de deformação da flutuação das velocidades. O espectro da velocidade longitudinal em função da frequência $f$ é definido como

$$
\mathrm{G}_{u u}(f)=2 \int_{-\infty}^{+\infty} C_{u u}(\tau) e^{-2 \pi i f \tau} d \tau
$$

o que corresponde à transformada de Fourier da função de auto-covariância $C_{u u}(\tau)$ ( $\tau$ é a separação no tempo) de uma série estacionária das flutuações da velocidade do vento longitudinal $u$. Segundo a teoria de Kolmogorov, existe uma faixa inercial em que $\mathrm{G}_{u u}$ e $\varepsilon$ são relacionados por (utilizando a hipótese da turbulência congelada de Taylor)

$$
\mathrm{G}_{u u}(f)=\left(\frac{2 \pi}{\bar{u}}\right)^{-2 / 3} \alpha_{1} \varepsilon^{2 / 3} f^{-5 / 3},
$$

onde $\bar{u}$ é a velocidade longitudinal média e $\alpha_{1}=0,4909$ é a constante de Kolmogorov (Chamecki e Dias, 2004). Já a ogiva é definida como (Dias, 2017)

$$
\operatorname{Og}_{u u}(f)=\int_{f}^{\infty} \mathrm{G}_{u u}\left(f^{\prime}\right) d f^{\prime}
$$

o que, para a faixa inercial, corresponde a

$$
\mathrm{Og}_{u u}(f)=\frac{3}{2}\left(\frac{2 \pi}{\bar{u}}\right)^{-2 / 3} \alpha_{1} \varepsilon^{2 / 3} f^{-2 / 3}=\frac{3}{2} \mathrm{G}_{u u}(f) f
$$

quando se considera que a faixa inercial se estende até infinito. 
Partindo das mesmas hipóteses, a função estrutura longitudinal de segunda ordem $\mathrm{D}_{11}\left(r_{1}\right)=\left\langle\left[u\left(x+r_{1}\right)-u(x)\right]^{2}\right\rangle$ na região inercial pode ser escrita como ( $r_{1}$ é a separação longitudinal e \langle\rangle é a média na direção longitudinal $\left.x\right)$

$$
\mathrm{D}_{11}\left(r_{1}\right)=0,4017 \alpha_{1} \varepsilon^{2 / 3} r_{1}^{2 / 3}
$$

e se relaciona com o espectro através de (utilizando o número de onda $k=2 \pi / f$ )

$$
\mathrm{D}_{11}\left(r_{1}\right)=\frac{4}{3} \int_{0}^{\infty} \mathrm{G}_{u u}(k) H\left(k r_{1}\right) d k, \quad H(\xi)=1+\frac{3 \cos (\xi)}{\xi^{2}}-\frac{3 \sin (\xi)}{\xi^{3}} .
$$

A Figura 1 apresenta $\mathrm{G}_{u u}(f), \mathrm{Og}_{u u}(f)$ e $\mathrm{D}_{11}\left(r_{1}\right)$ para $\bar{u}=0,5 \mathrm{~m} \mathrm{~s}^{-1}, \varepsilon=1 \times 10^{-4} \mathrm{~m}^{3} \mathrm{~s}^{-2}$ e valores de $5,56 \times 10^{-4} \leq$ $f \leq 10 \mathrm{~Hz}$ e $0,3 \leq r_{1} \leq 100 \mathrm{~m}$, intervalos correspondentes aos obtidos a partir de 30 minutos de dados medidos a $20 \mathrm{~Hz}$ (valores típicos das medições na camada superficial da atmosfera). As funções compensadas $f^{5 / 3} \mathrm{G}_{u u}(f), f^{2 / 3} \mathrm{Og}_{u u}(f)$ e $r_{1}^{-2 / 3} \mathrm{D}_{11}\left(r_{1}\right)$ (Fig. 1-(b) e (d)) são constantes proporcionais a $\varepsilon$, e por isso podem ser utilizadas para a sua estimação.
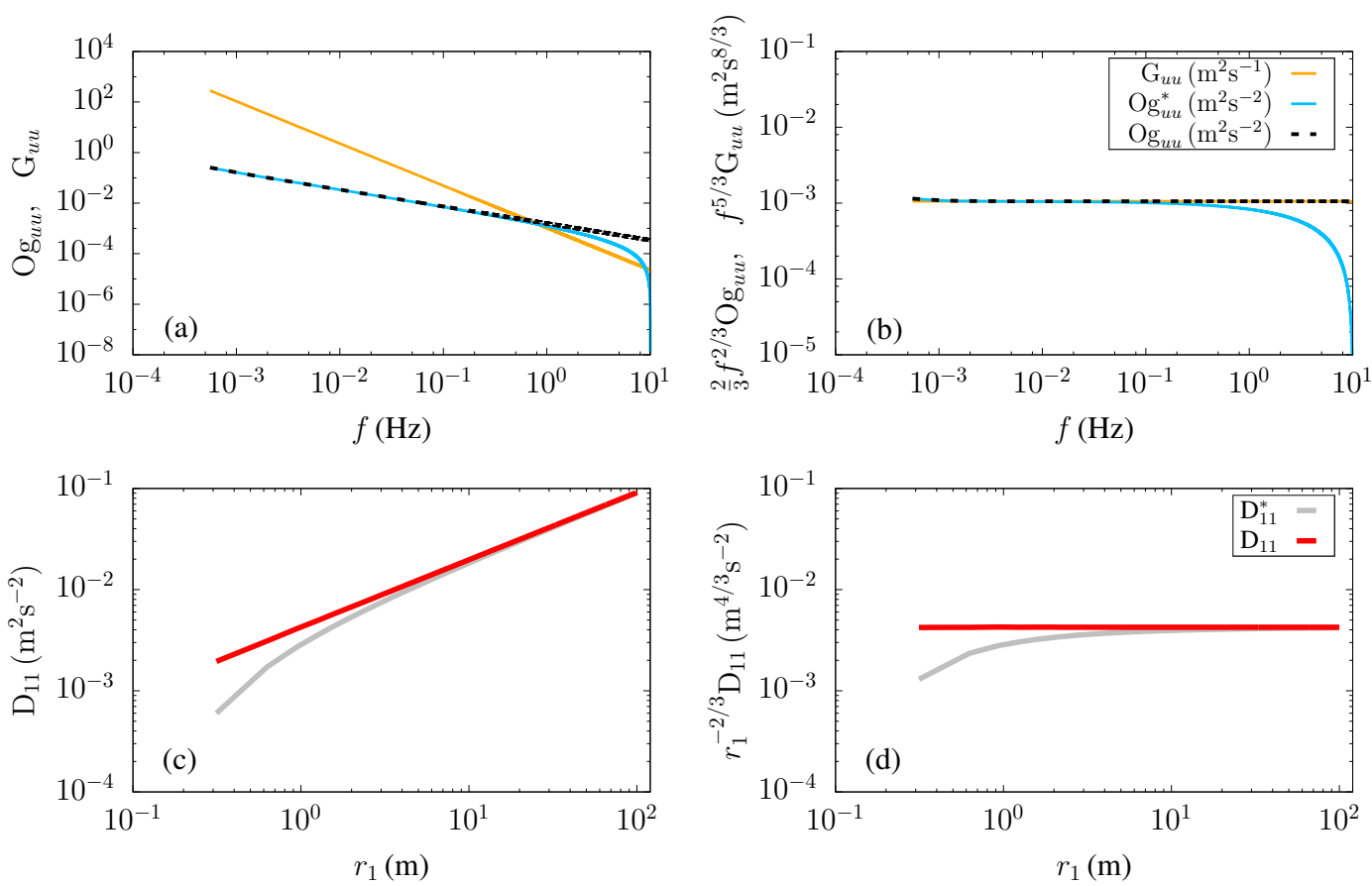

Figura 1: (a) Modelos teóricos e (b) modelos teóricos compensados do espectro (laranja) e ogiva (sem correção em azul, com correção em preto) na região inercial. (c) Modelo teórico e (d) modelo teórico compensado da função estrutura longitudinal de segunda ordem (sem correção em cinza, com correção em vermelho).

\section{Estimativa da ogiva e função estrutura a partir do espectro}

A ogiva e a função estrutura obtidas a partir da integração numérica de $\mathrm{G}_{u u}(f)$ até a frequência de $\mathrm{Nyquist}_{N y}=10 \mathrm{~Hz}\left(\mathrm{Og}_{u u}^{*}\right.$ e $\mathrm{D}_{11}^{*}\left(r_{1}\right)$ ) possuem uma subestimação numérica devido ao limite superior ser infinito na teoria (Fig. 1). Isso pode ser corrigido somando-se as elas o valor faltante da integral, o que corresponde a

$$
\int_{f_{N y}}^{\infty} \mathrm{G}_{u u}(f) d f=\frac{3}{2}\left(\frac{2 \pi}{\bar{u}}\right)^{-2 / 3} \alpha_{1} \varepsilon^{2 / 3} f_{N y}^{-2 / 3}
$$

para a ogiva e

$$
\int_{f_{N y}}^{\infty} \mathrm{G}_{u u}(k) H\left(k r_{1}\right) d k
$$

para a função estrutura. Em ambos os casos, ao ser utilizada a correção, recupera-se o valor teórico (Eqs. (4) e (5), ver Fig. 1-(a) e (c)). A estimativa de $\varepsilon$ através da média dos valores compensados da ogiva e função estrutura corrigidos (Fig. 1-(b) e (d)) retorna o valor correto. 


\section{Espectros sintéticos}

Para a avaliação dos erros associados à estimativa de $\varepsilon$ a partir do espectro, ogiva ou função estrutura, foram gerados, via método Monte Carlo, espectros sintéticos $G_{u u}^{s}$ que simulam erros aleatórios característicos de dados experimentais. Para isso, foram consideradas duas propriedades: (i) os espectros seguem uma distribuição qui-quadrado, já que correspondem à função de auto-covariância de uma variável com erro aleatório que segue uma distribuição normal de média zero e desvio padrão um; e (ii) os espectros possuem desvio padrão $\sigma_{\mathrm{G}}$ igual ao seu valor esperado (aqui correspondente ao espectro teórico de Kolmogorov $\mathrm{G}_{u u}$ ) (Dias, 2017). Portanto, para construir uma série sintética que segue uma distribuição qui-quadrado com média e desvio padrão igual à equação teórica do espectro (Eq. (2)), foi definido

$$
\begin{aligned}
\mathrm{G}_{u u}^{s}(f) & =\mathrm{G}_{u u}(f)+\sigma_{\mathrm{G}}(f) e_{\mathrm{G}}=\mathrm{G}_{u u}(f)\left(1+e_{\mathrm{G}}\right)=\left(\frac{2 \pi}{\bar{u}}\right)^{-2 / 3} \alpha_{1} \varepsilon^{2 / 3} f^{-5 / 3}\left(1+e_{\mathrm{G}}\right), \\
e_{\mathrm{G}} & \sim \frac{\chi^{2}(2)}{2}-1,
\end{aligned}
$$

onde $e_{\mathrm{G}}$ é o erro aleatório do espectro sintético, e $\chi^{2}(2)$ é a distribuição qui-quadrado com 2 graus de liberdade, que possui média e desvio padrão igual a 2 por definição. A Eq. (10) foi definida de forma a gerar um erro com média zero e desvio padrão 1.

\section{Ogiva e função estrutura sintéticas e a estimativa do erro da taxa de dissipação}

Após a geração de espectros sintéticos, a ogiva e função estrutura correspondentes foram obtidas a partir das Eqs. (3) e (6), através de uma integração numérica utilizando o método da quadratura de Gauss-Laguerre. Após a integração, os fatores de correção (Eqs. (7) e (8)) foram somados às ogivas e funções estrutura sintéticas. Por fim, a taxa de dissipação foi estimada a partir dos seus valores médios compensados nos intervalos $0,3 \leq f \leq 3 \mathrm{~Hz}$ e $1 \leq r_{1} \leq 10 \mathrm{~m}$ (intervalo aproximado da faixa inercial em dados experimentais, veja Figura 2 na próxima seção), ou seja

$$
\begin{aligned}
\varepsilon_{\mathrm{G}} & =\left(\frac{2 \pi}{\bar{u}}\right)\left(\frac{1}{\alpha_{1}} \overline{\mathrm{G}_{u u} f^{5 / 3}}\right)^{3 / 2}, \\
\varepsilon_{\mathrm{Og}} & =\frac{\left(\frac{2 \pi}{\bar{u}}\right)\left[\frac{2}{3 \alpha_{1}} \overline{\left.\left(\frac{\mathrm{Og}_{u u}^{*}}{f^{-2 / 3}-f_{N y}^{-2 / 3}}\right)\right]^{3 / 2}}\right.}{\varepsilon_{\mathrm{D}}}= \\
& \left.=\frac{\mathrm{D}_{11}^{*}}{0,4017 \alpha_{1} r_{1}^{2 / 3}-\mathrm{cor}}\right)^{3 / 2}
\end{aligned}
$$

onde cor $=\varepsilon^{-2 / 3} \times$ Eq. (8) (observe que a integral não possui solução analítica), e a barra superior representa a média aritmética.

Em dados experimentais, é comum que seja realizada uma suavização do espectro para redução dos erros aleatórios, através do uso de uma média móvel no campo da frequência. Para testar o efeito da suavização na estimativa de $\varepsilon$, uma média móvel com janela de 100 pontos foi aplicada nos espectros sintéticos, gerando uma quarta opção de estimativa da taxa de dissipação testada no presente estudo.

Ao todo, mil espectros foram simulados utilizando o método Monte Carlo, e estimativas de viés e erro médio quadrático para $\varepsilon_{\mathrm{G}}$ (com e sem filtro), $\varepsilon_{\mathrm{Og}}$ e $\varepsilon_{\mathrm{D}}$ foram computadas comparando-as com valor de $\varepsilon$ pré-definido.

\section{Resultados}

As Figuras 2-b e 2-d apresentam um exemplo de espectro sintético obtigo a partir da Eq. (9), cujo comportamento da faixa inercial em termos de intensidade e variabilidade das flutuações é comparável ao de um espectro obtido a partir de uma série de dados reais (Fig. 2-(a) e (c)). Note que, no espectro real, a faixa inercial tem aproximadamente uma década de tamanho, já que nas baixas frequências há uma região de produção de energia e nas altas frequências há um aumento de energia devido ao efeito de aliasing, ambos os efeitos típicos de turbulência atmosférica que estão fora do escopo do presente estudo.

A Tabela 1 apresenta os valores da média e da raiz do erro médio quadrático das estimativas de $\varepsilon$ para os mil espectros sintéticos gerados utilizando $\varepsilon=1 \times 10^{-4} \mathrm{~m}^{2} \mathrm{~s}^{-3}$. O efeito da suavização do espectro na estimativa de $\varepsilon$ é desprezível, visto que tanto na estimativa de $\varepsilon$ como na suavização do espectro é realizada uma média em $f$, o que torna o efeito da suavização redundante.

A Figura 3 apresenta cada uma das mil estimativas de $\varepsilon$ a partir do espectro e ogiva. A média das estimativas utilizando o espectro é muito próxima ao valor pré-definido (erro de 0,05\%). A estimativa utilizando a ogiva sem a correção possui um viés de 

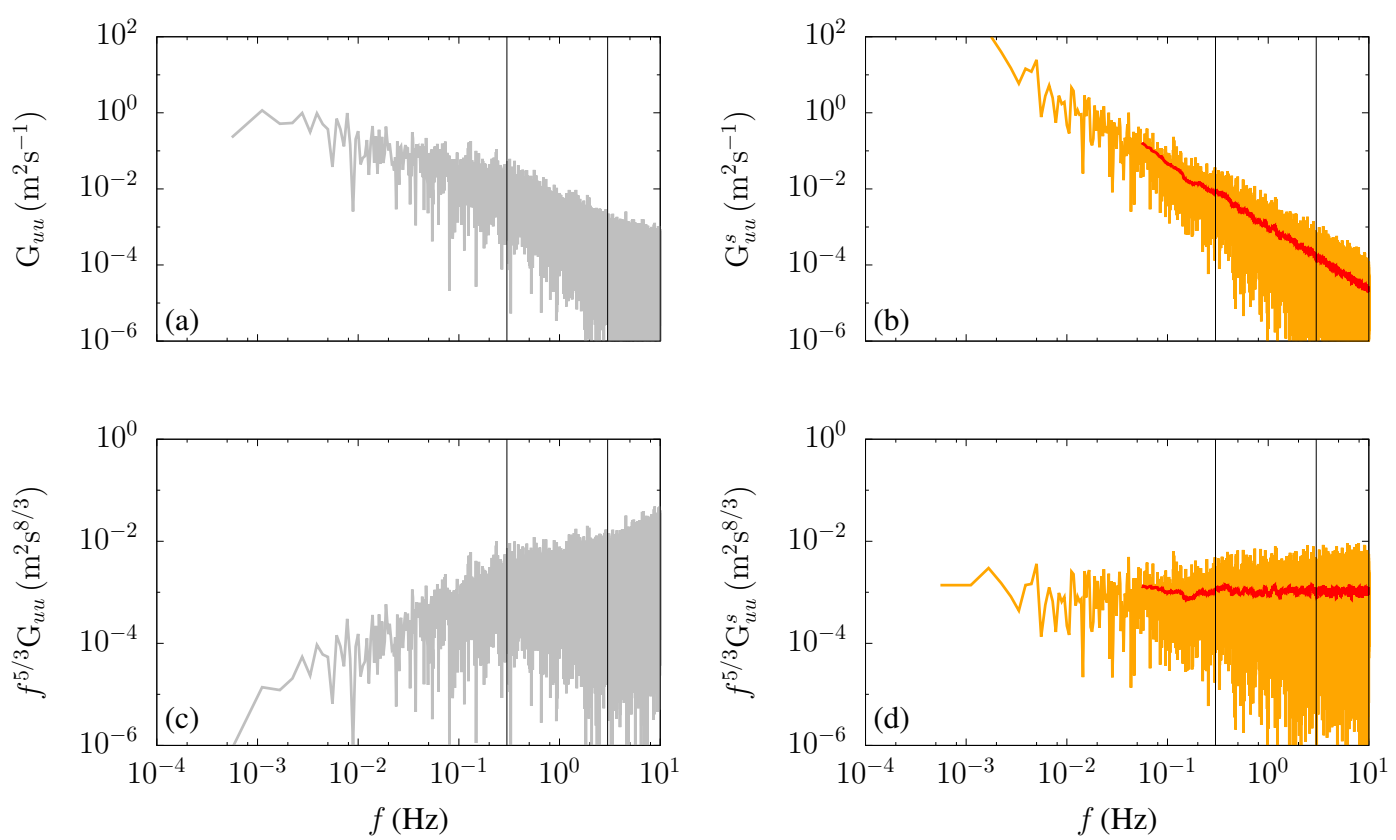

Figura 2: (a) Espectro e (c) espectro compensado da velocidade longitudinal medidos a $20 \mathrm{~Hz}$ durante um intervalo de 30 min a 2,92 m acima de uma superfície aquática (Dias, 2017). Exemplo de (b) espectro sintético e (d) espectro sintético compensado obtido a partir da Eq. (9) (em laranja). Em (b) e (d), o espectro suavizado é apresentado em vermelho. As linhas verticais demarcam a faixa inercial utilizada no presente estudo.

Tabela 1: Valores da média e da raiz do erro médio quadrático das estimativas de $\varepsilon=1 \times 10^{-4} \mathrm{~m}^{2} \mathrm{~s}^{-3}$ utilizando o espectro, a ogiva e a função estrutura. Ogiva e função estrutura obtidas a partir do espectro sem filtro.

\begin{tabular}{lcc}
\hline & média $\left(\mathrm{m}^{2} \mathrm{~s}^{-3}\right)$ & raiz do erro médio quadrático $\left(\mathrm{m}^{2} \mathrm{~s}^{-3}\right)$ \\
\hline espectro sem filtro & $1.0005 \times 10^{-4}$ & $2.1132 \times 10^{-6}$ \\
espectro com filtro & $1.0014 \times 10^{-4}$ & $2.1100 \times 10^{-6}$ \\
ogiva sem correção & $5.9550 \times 10^{-5}$ & $7.8664 \times 10^{-7}$ \\
ogiva com correção & $1.0002 \times 10^{-4}$ & $1.3080 \times 10^{-6}$ \\
função estrutura sem correção & $8.2078 \times 10^{-5}$ & $1.6449 \times 10^{-6}$ \\
função estrutura com correção & $1.0026 \times 10^{-4}$ & $1.9786 \times 10^{-6}$ \\
\hline
\end{tabular}

aproximadamente $-40 \%$, sendo que o viés é removido quando utilizada a correção (erro na ordem de $0,02 \%$ ). A raiz do erro médio quadrático da estimativa a partir da ogiva é aproximadamente $38 \%$ menor que o valor obtido na estimativa utilizando o espectro.

As estimativas da taxa de dissipação a partir da função estrutura são apresentadas na Figura 4. Assim como no caso da ogiva, a estimativa a partir da função estrutura apresenta um viés negativo (de aproximadamente - 18\%) que é removido quando utilizada a correção. Já a raiz do erro médio quadrático da estimativa a partir da função estrutura é aproximadamente $6 \%$ inferior ao valor da estimativa utilizando o espectro.

Por fim, é importante salientar que esses resultados são independentes do valor de $\varepsilon$ e $\bar{u}$ pré-definidos, porém eles dependem do tamanho da faixa inercial escolhida, já que o erro do espectro diminui com o aumento da faixa devido à presença de erros aleatórios em cada frequência, enquanto que os erros da ogiva e função estrutura são menos dependentes do tamanho da faixa já que $\mathrm{Og}_{u u}$ e $\mathrm{D}_{11}$ já correspondem a integrais do espectro em $f$ por definição (portanto o erro aleatório já está compensado). Os resultados apresentados nesse estudo correspondem aos erros obtidos quando utilizada uma faixa inercial de uma década, tamanho aproximado da faixa inercial presente em dados de campo (veja os dados reais da Figura 2 como exemplo).

\section{Conclusões}

As estimativas da taxa de dissipação da energia cinética da turbulência a partir de dados de velocidade longitudinal podem ser realizadas utilizando o espectro, a ogiva ou a função estrutura de segunda ordem. Quando utilizada a ogiva ou a função estrutura, é 

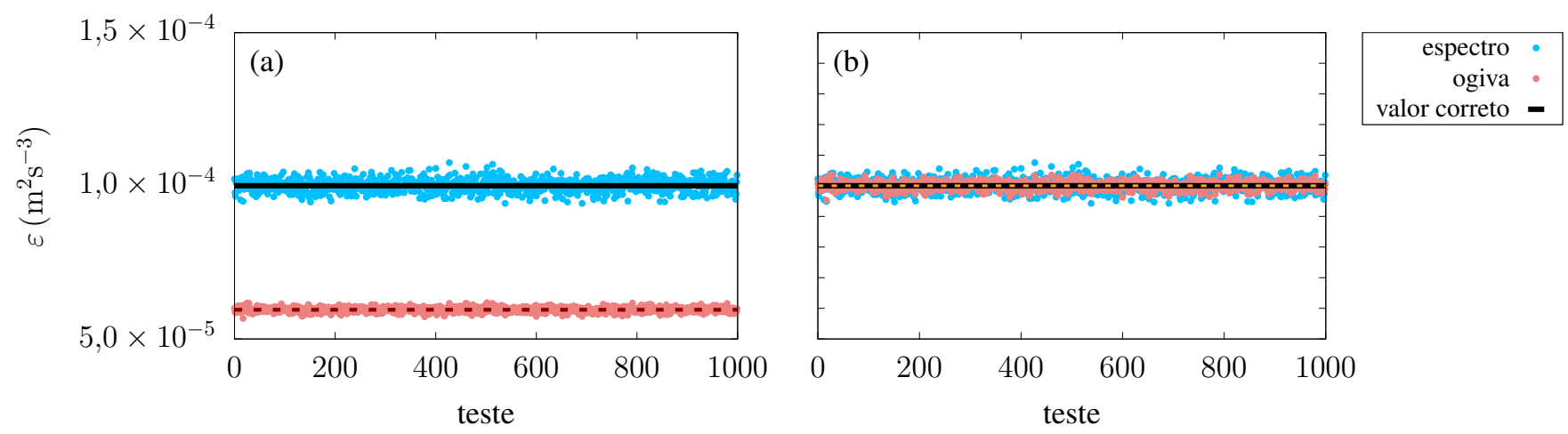

Figura 3: Taxa de dissipação da ECT pré-definida (linha preta) e estimada utilizando espectro (pontos azuis) e ogiva (pontos vermelhos), para cada um dos mil testes realizados. Estimativas a partir da ogiva (a) sem correção e (b) com correção. A linha tracejada representa a média das estimativas a partir da ogiva.
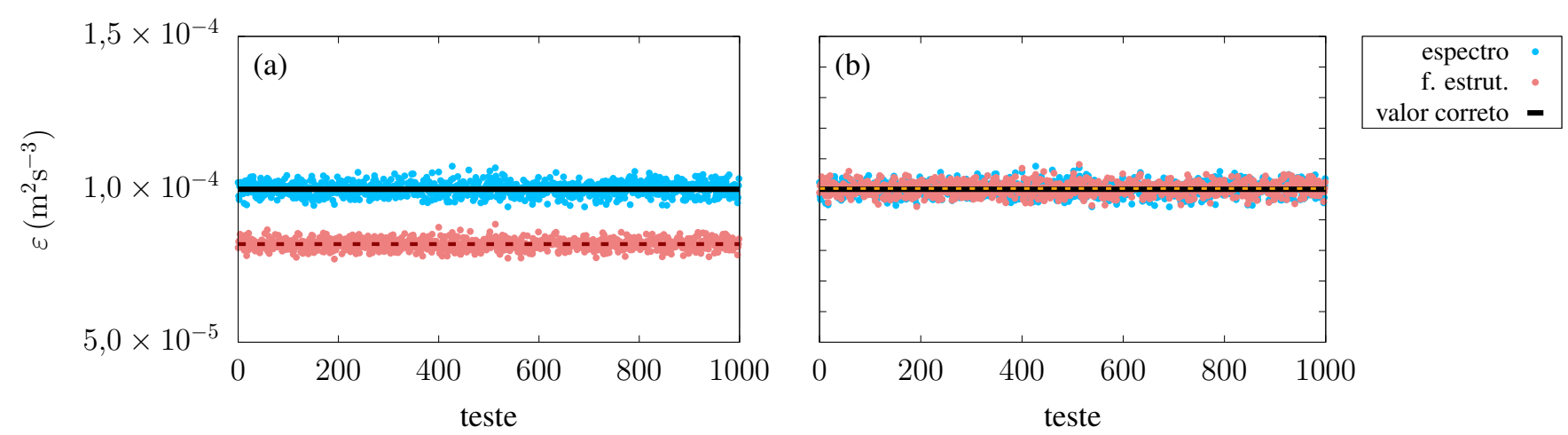

Figura 4: Taxa de dissipação da ECT pré-definida (linha preta) e estimada utilizando espectro (pontos azuis) e função estrutura (pontos vermelhos), para cada um dos mil testes realizados. Estimativas a partir da função estrutura (a) sem correção e (b) com correção. A linha tracejada representa a média das estimativas a partir da função estrutura.

necessário adicionar um fator de correção para remover o viés causado pela frequência de medição finita. A estimativa utilizando a ogiva deve ser priorizada já que ela apresenta o menor erro quando comparada às estimativas utilizando o espectro ou a função estrutura (na ordem de $1,3 \%$ do valor esperado).

\section{Referências}

Chamecki, M., Dias, N. L. (2004). The local isotropy hypothesis and the turbulent kinetic energy dissipation rate in the atmospheric surface layer. Quarterly Journal of the Royal Meteorological Society, 130(603), 2733-2752, URL http: / / dx . doi . org/ $10.1256 /$ qj.03.155.

Chamecki, M., Dias, N. L., Salesky, S. T., Pan, Y. (2017). Scaling laws for the longitudinal structure function in the atmospheric surface layer. Journal of the Atmospheric Sciences, 74(4), 1127-1147, URL https://doi .org/10.1175/ JAS-D-16-0228.1, https://doi.org/10.1175/JAS-D-16-0228.1.

Davidson, P. (2004). Turbulence, an Introduction for scientists and engineerers. Oxford University Press.

Davidson, P., Krogstad, P. A. (2014). A universal scaling for low-order structure functions in the log-law region of smoothand rough-wall boundary layers. Journal of Fluid Mechanics, 752, 140-156, URL http://dx. doi.org/10.1017/jfm. 2014.286.

Dias, N. L. (2017). Smoothed spectra, ogives, and error estimates for atmospheric turbulence data. Boundary-Layer Meteorology, (n/a), n/a-n/a, URL https://doi.org/10.1007/s10546-017-0293-7.

Katul, G., Mahrt, L., Poggi, D., Sanz, C. (2004). One and two-equation models for canopy turbulence. Boundary-Layer Meteorology, 113(1), 81-109, URL http://dx.doi.org/10.1023/B:BOUN.0000037333.48760.e5. 
Moeng, C. H., Wyngaard, J. C. (1989). Evaluation of turbulent transport and dissipation closures in second-order modeling. Journal of the Atmospheric Sciences, 46(14), 2311-2330, URL http://dx. doi .org/10.1175/1520-0469 (1989) $046<2311$ : EOTTAD>2.0.CO; 2, http://dx. doi.org/10.1175/1520-0469 (1989) 046<2311:EOTTAD>2. $0 . \mathrm{CO} ; 2$.

Pan, Y., Chamecki, M. (2016). A scaling law for the shear-production range of second-order structure functions. Journal of Fluid Mechanics, 801,459-474, URL http://dx.doi.org/10.1017/jfm.2016.427.

\section{Lívia Souza Freire}

Nelson Luís Dias

\section{André Luiz Grion}

Universidade Federal do Paraná , Brasil E-mail: liviagrion@gmail.com

Universidade Federal do Paraná, Brasil

E-mail: nldias@ufpr.br

Universidade Federal do Paraná , Brasil E-mail: grion@ufpr.br 